\title{
Surface plasmon enhanced light emission from CdSe quantum dot nanocrystals
}

\author{
Koichi Okamoto, Saurabh Vyawahare, and Axel Scherer \\ Department of Applied Physics and Physics, California Institute of Technology, MC 136-93, Pasadena, CA91125
}

\begin{abstract}
We obtained very bright light emission from CdSe quantum dots (QDs) by using surface plasmon coupling. Both the photoluminescence intensities and decay rates are dramatically increased when QDs are located on evaporated gold films. (C)2000 Optical Society of America

OCIS codes: (240.6680) Surface plasmons; (250.5230); (300.6500) Spectroscopy, time-resolved
\end{abstract}

Currently, solid-state light emitting devices have been developed and expected to eventually replace more traditional fluorescent tubes as illumination sources. However, their light emission efficiencies are still substantially lower than those of fluorescent tubes. The most important requirement for a competitive solid-state light emitting device is to increase its quantum efficiency of light emission. Recently, we have reported a method for enhancing the light emission efficiency from InGaN quantum well (QW) by controlling the energy transfer between QW and surface plasmon (SP). [1] We have directly measured significant enhancement of internal quantum efficiency of emission due to spontaneous recombination rate increases. [2] We believe that this SP enhanced technique can be applied not only to InGaN-based materials but also to various materials that suffer from low quantum efficiencies. Indeed, we have also reported the similar light enhancement for dye molecule doped polymer materials. [3] Semiconductor quantum dot (QD) nanocrystals are one of the very promising materials for light emitting source. QD nanocrystals possess a number of advantageous features such as light emitters, solar cells, and fluorescent labels. [4] In this study, we investigate the direct observation of SP coupled spontaneous emission from CdSe and CdSe/ZnS QDs by using the time-resolved photoluminescence (PL) measurements and consider the mechanisms and dynamics of the surface plasmon coupling and the light emission enhancement.

CdSe-based QDs were purchased from Evident Technologies. These QDs have emission peak around $620 \mathrm{~nm}$ and the crystal diameter is around $5 \mathrm{~nm}$. The toluene solutions of the QDs were dispersed on quartz substrates. After the solutions evaporated, monolayers of the QD nanocrystals remained on the substrates. The half parts of the quartz surface were covered by $50 \mathrm{~nm}$ gold layer by thermal evaporation. To perform time-resolved PL measurements, a frequency doubled beam of a mode-locked Ti: sapphire laser (Spectra Physics) was used to excite the samples. The pulse width, wavelength, and repetition rate were chosen as $100 \mathrm{fs}, 400 \mathrm{~nm}$, and $80 \mathrm{MHz}$, respectively. A Hamamatsu Photonics C4334 Streak Camera system with a time resolution of 5 ps served as the detector.

Fig. 1(a) shows the PL spectrum taken for the CdSe QDs. As the PL peak of the QDs on quartz was normalized to 1, it is clear that a dramatic enhancement in the PL intensity from the QDs on gold layer. The obtained over 20fold increasing of PL intensity should be attributed to strong interaction with SP. The electron-hole pairs generated in the QDs couple to electron vibrations at the gold surface when the energies of electron-hole pairs in the QDs and of the metal SPs are similar. Fig. 1(b) show the PL time-profile of the CdSe QDs on gold and quartz. Clearly, the PL
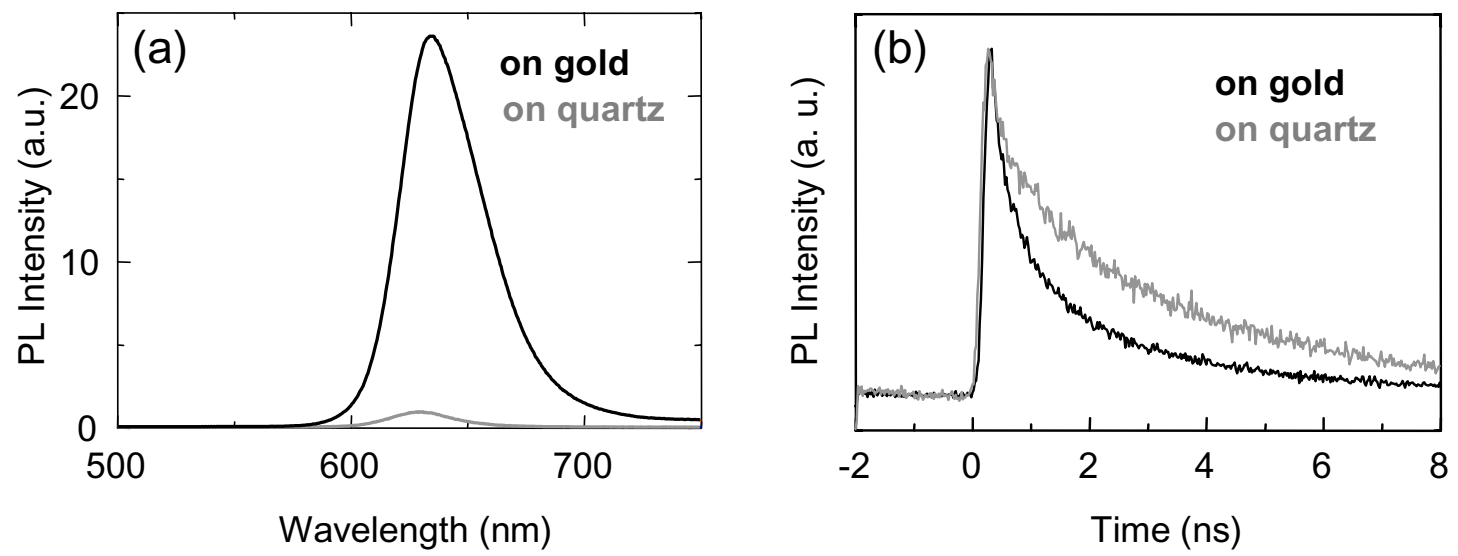

Fig. 1 (a) Photoluminescence (PL) spectra and (b) PL time-profiles of CdSe nanocrystals on gold and on quartz. 


\section{QTuD3.pdf}

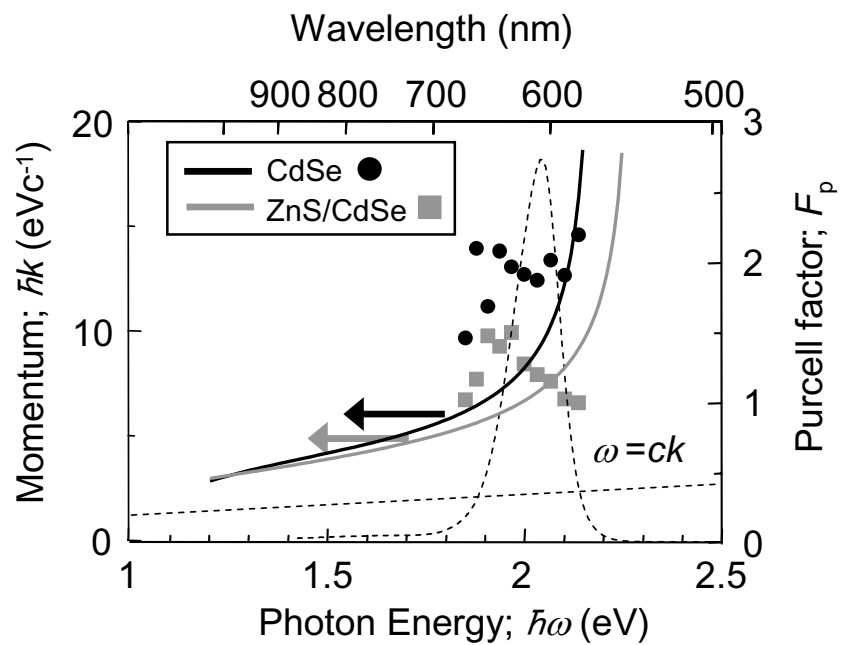

Fig. 2 Dispersion diagram of surface plasmon mode for CdSe and CdSe/ZnS with gold. Purcell enhancement factors obtained by the ratios of PL lifetimes were also plotted against wavelength.

decay of QDs on gold is faster than that of QDs on quartz. This suggests that the PL decay rates are also enhanced by the SP coupling. This should happen if the QD-SP coupling rate is much faster than the radiative and nonradiative recombination rates of the electron-hole pairs. High electromagnetic fields are introduced by the large density of states from the SP dispersion diagram, and these fields increases the QD-SP coupling rate. If the metal surface is perfectly flat, the SP energy would be thermally dissipated. However, the SP energy can be extracted as light by providing roughness or nano-structuring the metal layer. Such roughness allows SPs of high momentum to scatter, lose momentum and couple to radiated light. The few tens of nanometer sized roughness in the gold surface layer can be obtained by controlling the evaporation conditions. The roughness of the gold surface was actually observed by the atomic force microscopy and the scanning electron microscopy. We investigated the similar measurements for CdSe cores with $\mathrm{ZnS}$ shells, but the enhancements of both PL intensity and PL decay rate were not so remarkable compared to the results of naked CdSe without shells. Elucidating the reason of this discrepancy should be useful to understand the surface plasmon coupling mechanism and to optimize more efficient coupling conditions.

The obtained PL time-profiles were able to be fitted to single exponential functions and the PL decay lifetimes were obtained. The Purcell enhancement factor $\left(F_{\mathrm{p}}\right)$ quantifies the increase in spontaneous emission rate into a mode of interest, and be obtained by the ratios of the original and enhanced PL lifetimes. The obtained $F_{\mathrm{p}}$ values of CdSe and $\mathrm{CdSe} / \mathrm{ZnS}$ QDs were plotted against wavelength in Fig. 2. The black and gray curves in Fig. 2 are the dispersion diagram of SP mode calculated with dielectric function of $\mathrm{Au} / \mathrm{CdSe}$ and $\mathrm{Au} / \mathrm{ZnS}$, respectively. The wavelength dependence behavior of obtained $F_{\mathrm{p}}$ is correlated to the dispersion diagram. The SP frequency of $\mathrm{Au} / \mathrm{CdSe}$ is well matched to the emission spectrum of QDs (dashed line) especially at the higher energy region. The obtained $F_{\mathrm{p}}$ values of CdSe are also increased at the higher energy region. On the other hand, the SP frequency of $\mathrm{Au} / \mathrm{ZnS}$ is slightly far from the emission spectrum. This should be reason of the discrepancy of the SP enchantment for CdSe and $\mathrm{CdSe} / \mathrm{ZnS}$ QDs with gold layer. This clear correlation supports our proposed QD-SP coupling mechanism.

SP coupling is one of the most interesting methods for developing efficient solid-state light emitting devices, as the metal can be used both as electrical contact and for exciting plasmons. We observed dramatically enhancement of both the PL intensities and decay when QDs are located on evaporated gold films. By using this technique, highspeed and efficient light emission is predicted for optically as well as electrically pumped light emitters, because the mechanism should be not related to the pumping method.

[1] K. Okamoto, I. Niki, A. Shvartser, Y. Narukawa, T. Mukai, and A. Scherer, "Surface plasmon enhanced light-emitters based on InGaN quantum wells", Nature Materials, 3, 601-605 (2004).

[2] K. Okamoto, I. Niki, A. Scherer, Y. Narukawa, T. Mukai, Y. Kawakami, "Surface plasmon enhanced spontaneous emission rate of InGaN/GaN quantum wells probed by time-resolved photoluminescence spectroscopy", Appl. Phys. Lett., 87, 071102 (2005).

[3] T. D. Neal, K. Okamoto, A. Scherer, "Surface plasmon enhanced emission from dye doped polymer layers", Optics Express, 13, 5522-5527 (2005).

[4] M. Bruchez Jr., M. Moronne, P. Gin, S. Weiss, and A. P. Alivisatos, "Semiconductor nanocrystals as fluorescent biological labels", Science 281, 2013-2016 (1998) 